\title{
Microsurgical clipping of middle cerebral artery aneurysms: preoperative planning using virtual reality to reduce procedure time
}

\author{
Thomas C. Steineke, MD, PhD, ${ }^{1}$ and Daniela Barbery, BS ${ }^{2}$ \\ ${ }^{1}$ Neurosurgery, JFK Neuroscience Institute at Hackensack Meridian Health JFK University Medical Center, Edison, New Jersey; \\ and ${ }^{2}$ Surgical Theater, Inc., Cleveland, Ohio
}

OBJECTIVE The authors sought to evaluate the impact of virtual reality (VR) applications for preoperative planning and rehearsal on the total procedure time of microsurgical clipping of middle cerebral artery (MCA) ruptured and unruptured aneurysms compared with standard surgical planning.

METHODS A retrospective review of 21 patients from 2016 to 2019 was conducted to determine the impact on the procedure time of MCA aneurysm clipping after implementing VR for preoperative planning and rehearsal. The control group consisted of patients whose procedures were planned with standard CTA and DSA scans $(n=11)$. The VR group consisted of patients whose procedures were planned with a patient-specific $360^{\circ} \mathrm{VR}(360 \mathrm{VR})$ model $(n=10)$. The 360VR model was rendered using CTA and DSA data when available. Each patient was analyzed and scored with a case complexity (CC) 5-point grading scale accounting for aneurysm size, incorporation of $\mathrm{M}_{2}$ branches, and aspect ratio, with 1 being the least complex and 5 being the most complex. The mean procedure times were compared between the VR group and the control group, as were the mean CC score between the groups. Comorbidities and aneurysm conduction (ruptured vs unruptured) were also taken into consideration for the comparison.

RESULTS The mean CC scores for the control group and VR group were $2.45 \pm 1.13$ and $2.30 \pm 0.48$, respectively. $C C$ was not significantly different between the two groups $(p=0.69)$. The mean procedure time was significantly lower for the VR group compared with the control group ( 247.80 minutes vs 328.27 minutes; $p=0.0115$ ), particularly for the patients with a CC score of $2(95 \% \mathrm{Cl}, p=0.0064)$. A Charlson Comorbidity Index score was also calculated for each group, but no statistical significance was found (VR group, 2.8 vs control group, 1.8, $p=0.14$ ).

CONCLUSIONS In this study, usage of $360 \mathrm{VR}$ models for planning the craniotomy and rehearsing with various clip sizes and configurations resulted in an 80 -minute decrease in procedure time. These findings have suggested the potential of VR technology in improving surgical efficiency for aneurysm clipping procedures regardless of complexity, while making the procedure faster and safer.

https://thejns.org/doi/abs/10.3171/2021.5.FOCUS21238

KEYWORDS virtual reality; middle cerebral artery aneurysm; microsurgical clipping; craniotomy planning

$\mathrm{A}$ MIDDLE cerebral artery (MCA) aneurysm is relatively common, with a $15 \%$ to $43 \%$ higher incidence rate compared with other cerebral aneurysms. ${ }^{1-3}$ While endovascular embolization with coils is a popular management option for MCA aneurysms, studies have indicated a trend of better clinical outcomes and fewer associated risks with microsurgical clipping in this location. ${ }^{4,5}$ Thus, microsurgical clipping remains the preferred treatment approach for MCA aneurysms. ${ }^{6}$ The standard surgical approach for treating MCA aneurysms is a pterional craniotomy; however, the field is trending toward minimally invasive surgery techniques with the help of virtual reality (VR) technology. Several groups have implemented presurgical VR simulation to plan and execute more individualized and less-invasive craniotomies for aneurysm clipping procedures. ${ }^{7-9}$ In one study, the authors used VR-aided craniotomy management planning to measure and mark the distances from specific anatomical landmarks to the planned craniotomy with a ruler and marker onto the patient's head. This technique enabled surgeons to correctly place the craniotomy in $98 \%$ of cases including ones during which neuronavigation failed $(\mathrm{n}=48))^{7}$ Another study utilized VR for crani-

ABBREVIATIONS CC = case complexity; $\mathrm{CCI}=$ Charlson Comorbidity Index; $\mathrm{CVA}=$ cerebrovascular accident; EBL = estimated blood loss; LOC = level of consciousness; $\mathrm{MCA}=$ middle cerebral artery; $\mathrm{mRS}=$ modified Rankin Scale; $\mathrm{OR}=$ operating room; $\mathrm{SAH}=$ subarachnoid hemorrhage; VR = virtual reality; $360 \mathrm{VR}=360^{\circ} \mathrm{VR}$. SUBMITTED April 1, 2021. ACCEPTED May 13, 2021.

INCLUDE WHEN CITING DOI: 10.3171/2021.5.FOCUS21238. 


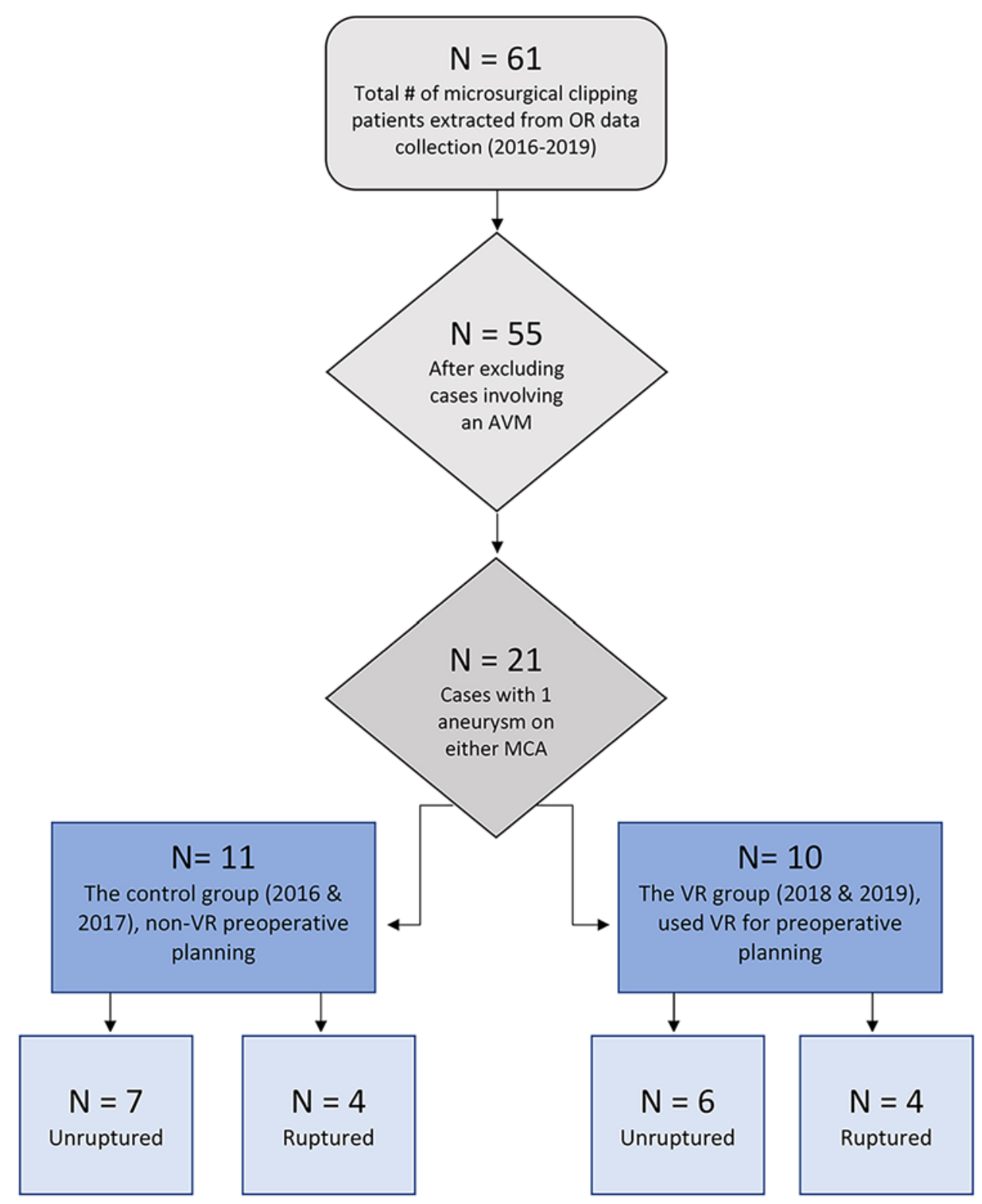

FIG. 1. Flow chart outlining the retrospective review.

otomy planning, focusing on patient positioning to identify the optimal trajectory to the aneurysm. ${ }^{8}$

Developments in the software and hardware components of VR technology have facilitated its increased adoption in medicine. Specifically, VR has been explored for presurgical planning and rehearsal of aneurysm microsurgical clip occlusion using 3D reconstructions of the patient's preoperative scans in DICOM format. ${ }^{9-11} \mathrm{VR}$ allows for immersive surgical planning using a 3D-reconstructed model that combines all pertinent imaging modalities in one space to provide surgeons with a comprehensive view of the patient's unique anatomy. ${ }^{12-14}$ Preoperative planning in conjunction with virtual rehearsal of the planned procedure may help prepare the surgeon for each procedure and consequently increase surgical efficiency and performance.

Here, we evaluate the impact of preoperative planning and rehearsal in VR on procedure time, complication rates, length of stay, and estimated blood loss (EBL) associated with aneurysm clipping procedures. A VR visualization platform was employed to create patient-specific $360^{\circ}$ VR (360VR) models reconstructed from CTA and DSA scans, which were used for craniotomy planning and aneurysm clipping rehearsal for MCA aneurysms.

\section{Methods}

\section{Patient Selection}

A retrospective review of patients who underwent microsurgical clipping from 2016 to 2019 was conducted. Initially, the sample size consisted of 61 patients; only patients with a single aneurysm involving the MCA bifurcation were included $(n=21)$ (Fig. 1). All patients un- 

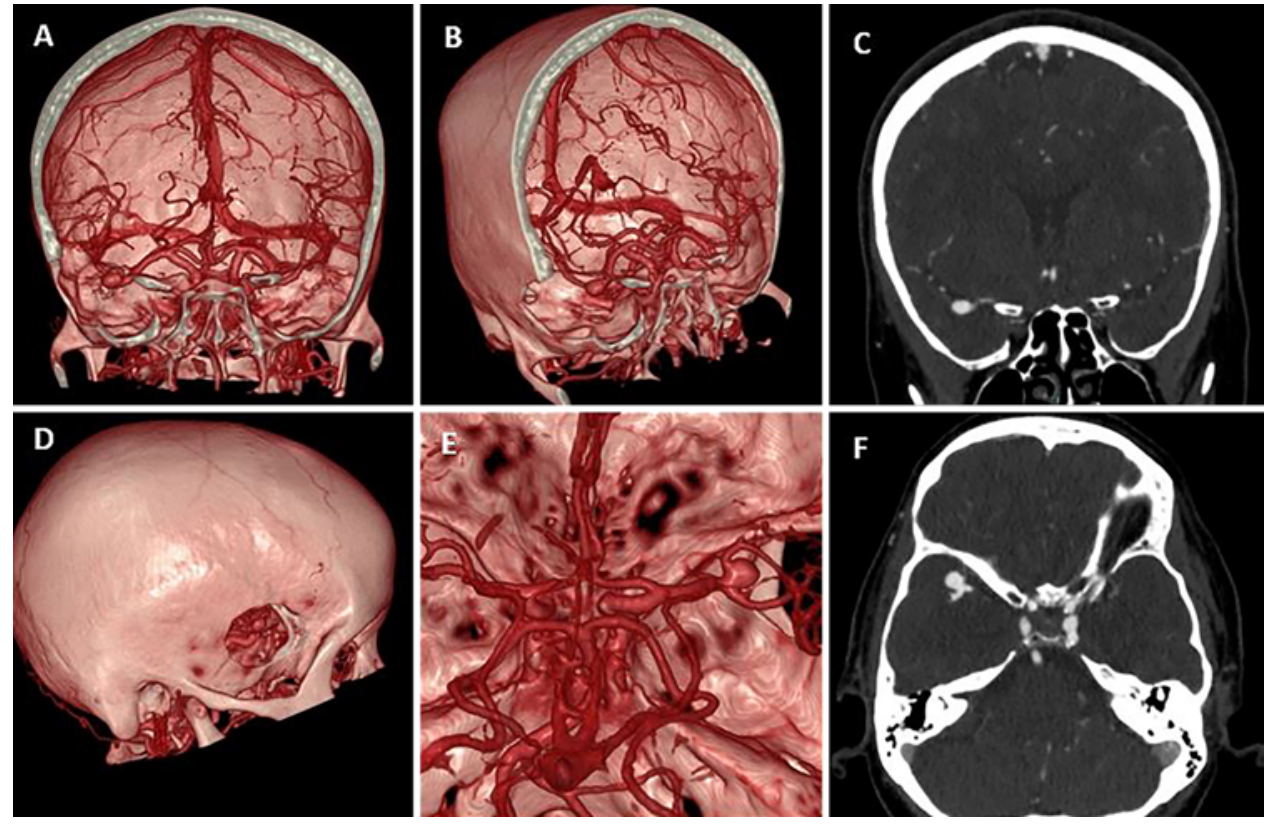

FIG. 2. Preoperative imaging of a right MCA aneurysm. A and B: Snapshots of the 360 VR model in the coronal view. C: Corresponding DICOM of a coronal CTA scan. D: Snapshot of sagittal view of the 360VR model with a virtual corridor placed to represent the surgeon's craniotomy and approach. E: Close-up of the model in axial view. F: Axial CTA DICOM scan.

derwent surgery in the same hospital and with the same expert neurosurgery attending who had 13 to 16 years of experience (T.C.S.). Patients from 2016 and 2017 received standard care (non-VR control group; $\mathrm{n}=11$ ). For patients from January 2018 through December 2019, VR was implemented for preoperative planning using patient-specific 360VR models $(\mathrm{n}=10)$.

\section{VR Case Building}

The 360VR models were created by reconstructing the patient's preoperative imaging using The SuRgical Planning platform (version 7.6.0, Surgical Theater, Inc.). Each case was built using patient-specific CTA scans and, when available, DSA scans (Fig. 2). These models required high-resolution volumetric scans that are established imaging protocols at this institution. The resolutions, defined as spacing between each slice in the axial plane, of the CTA and DSA scans were $0.64 \mathrm{~mm}$ and 0.34 $\mathrm{mm}$, respectively. If the patient had both CTA and DSA studies available, the images were fused together to enhance the critical vessels on the 360VR model. A trained technician took 5 to 15 minutes to render each case depending on the number of scans fused in a single model, which was later approved by the surgeon. Postoperatively, the surgeon ordered a 3-month follow-up CTA that was reconstructed and evaluated in VR to confirm that the clipping was successful.

\section{Preoperative Planning Workflow}

For the control group, standard CTA and DSA imaging studies were utilized exclusively for preoperative planning. Preoperative planning was conducted by carefully reviewing the CTA studies to better understand the shape and size of the aneurysm in the context of the skull and the sylvian fissure. Furthermore, 3D image reconstructions using both CTA and DSA were analyzed to examine the geometry of the aneurysm and potentially strategize which clips would be necessary in order to occlude the aneurysm. For the VR group, the patient's CTA and DSA scans were rendered into a patient-specific 360VR model that provided detailed visualization of the patient's pathology and surrounding anatomy. Preoperative planning in the VR group was conducted using the VR planning platform in the operating room (OR) while the patient was being prepped for surgery. The surgeon used the VR headset (Rift, Oculus) and controllers to plan the craniotomy and rehearse clipping with various clip sizes and configurations. Realistically rendered tools included in the VR platform were utilized. The virtual drill was used to assess craniotomy location and size, and virtual clips of different sizes were used to rehearse clipping at various angles. This allowed the surgeon to "practice" clipping the aneurysm using a variety of clips. Furthermore, we were disciplined to ensure that the angles were realistic and true to what would be encountered intraoperatively. Once the trajectory and clips were selected during the VR planning, the 360VR model was displayed in the OR near the surgical field for reference (Fig. 3). Given that the VR preoperative rehearsal was performed simultaneously during the OR setup or while anesthesia was being administered to the patient, no extra time was added to this workflow (Video 1).

VIDEO 1. Microsurgical clipping of a left MCA with preoperative planning and rehearsal. Copyright Thomas Steineke. Published with permission. Click here to view.

\section{Data Collection}

Procedure time, defined as the time of first incision to 

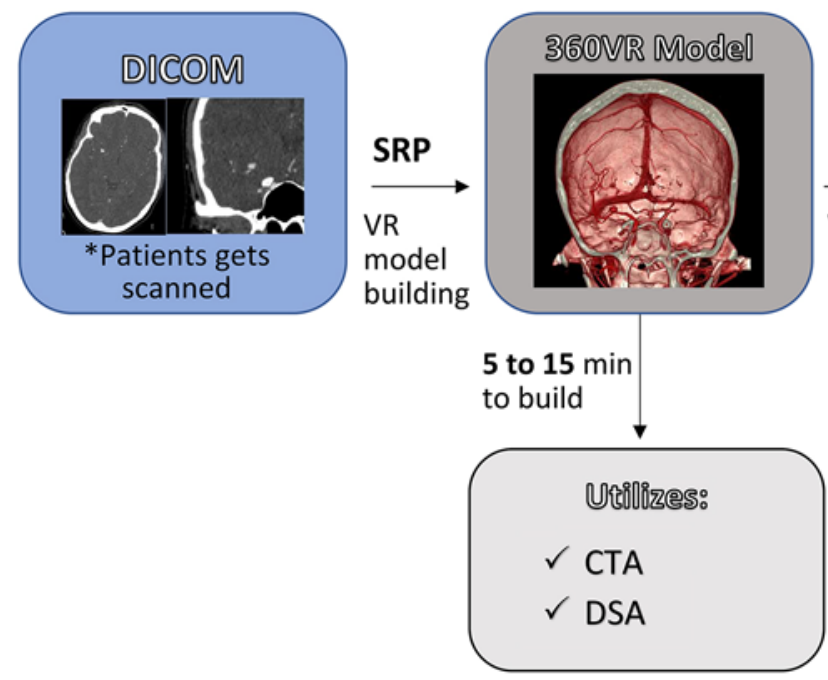

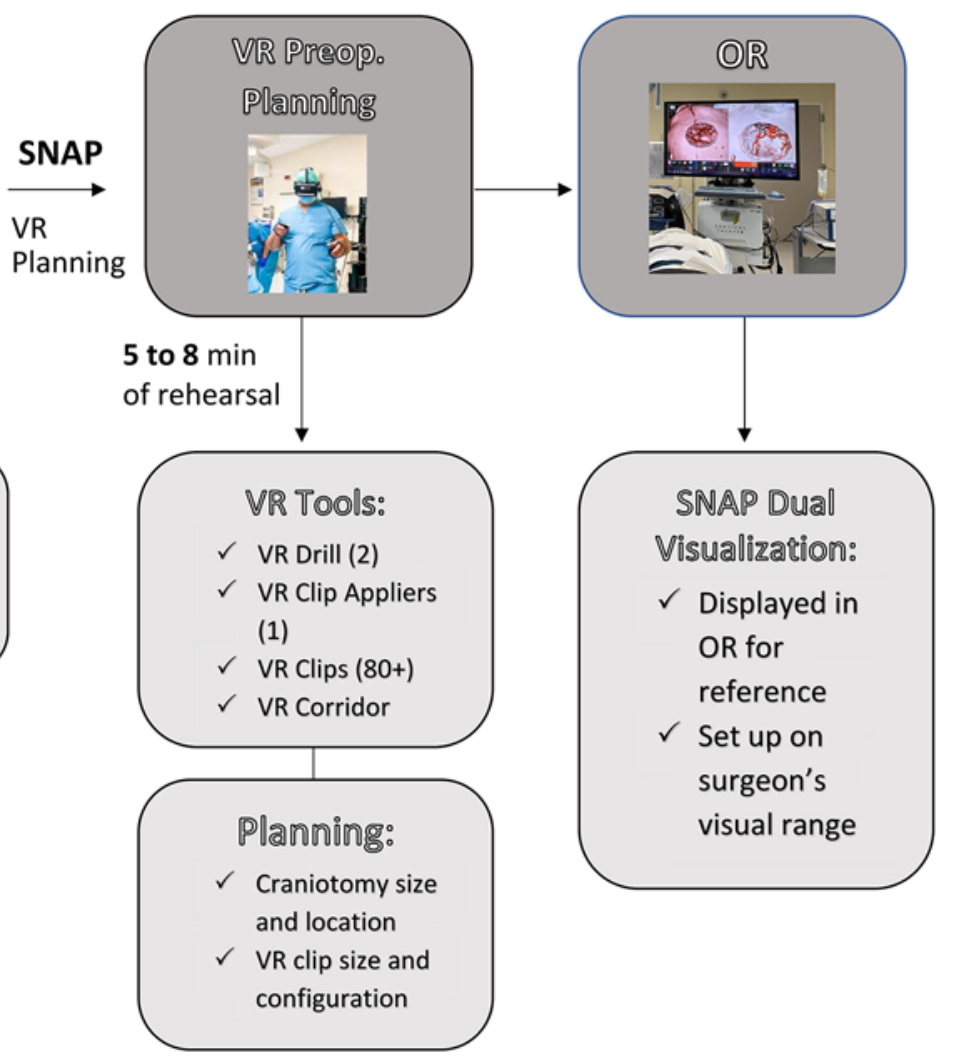

FIG. 3. Workflow for the aneurysm clipping procedure utilizing VR. The surgeon used the 360VR model rendered from DICOM images with the VR headset and controllers to plan and rehearse prior to scrubbing in for each surgery in the VR group. The model was used as a reference only in the OR using the Surgical Navigation Advanced Platform (SNAP).

the time of closing, was recorded for each patient. Patient charts were reviewed to collect basic demographics (e.g., sex, age), comorbidities, preoperative neurological deficits, hospital length of stay, estimated blood loss (EBL), and outcomes both immediately and 3 months postsurgery. This information was gathered to establish the general health of each group for comparison using the Charlson Comorbidity Index (CCI). ${ }^{15}$

\section{Case Complexity Scoring}

Case complexity (CC) was assessed based on aneurysm size, incorporation of distal branches, and the aspect ratio of the aneurysm. All 11 patients from the non-VR group were rendered into a $360 \mathrm{VR}$ model to maintain a consistent scoring method for both sample groups. The same neurosurgeon attending, blinded to the date of the scan, measured the aneurysms in VR and assessed their characteristics. The aneurysm size was scored based on the greatest diameter measurement on the XYZ plane $(<$ $5 \mathrm{~mm}=0$ points, $5-8 \mathrm{~mm}=1$ point, $9-12 \mathrm{~mm}=2$ points, and $>12 \mathrm{~mm}=3$ points). If the aneurysm contained any distal branches, 1 point was added to the total. The aspect ratio was calculated by dividing the aneurysm diameter of the dome by the width of the neck $(>1.6=0$ points and $<1.6=1$ point). These three components were selected by the surgeon to calculate a complexity scale from 1 to 5 , with 1 being the least complex and 5 being the most complex. This grading system was adapted from Etminan et al. and was an attempt to develop a score to reflect the difficulty of microsurgical treatment of any given MCA aneurysm. ${ }^{16}$ The rationale is that the difficulty of surgical treatment of an aneurysm generally increases with the size of the aneurysm, the aspect ratio (for an MCA aneurysm, a large aspect ratio typically means that the aneurysm incorporates the bifurcation of the MCA into the base of the aneurysm), and whether the $\mathrm{M}_{2}$ branches are incorporated into the aneurysm requiring reconstruction of the distal branches.

\section{Statistical Analysis}

Unpaired t-tests were performed to compare procedure times, CC scores, and CCI scores between the non-VR and VR groups, with $\mathrm{p} \leq 0.05$ considered statistically significant. These variables were calculated with $95 \%$ confidence intervals to evaluate if there was a significant difference in procedure time among the sample groups and if there were other elements that factored in.

\section{Results}

Table 1 summarizes patient demographics, including sex, age, and smoking status. For the non-VR control group, the mean procedure time and $\mathrm{CC}$ score were $328.27 \pm$ SD 73.86 minutes and $2.45 \pm 1.13$, respectively 
TABLE 1. Patient demographic summary for the non-VR and VR groups

\begin{tabular}{lccc}
\hline \multicolumn{1}{c}{ Demographics } & $\begin{array}{c}\text { Control Group } \\
(\mathrm{n}=11)\end{array}$ & $\begin{array}{c}\text { VR Group } \\
(\mathrm{n}=10)\end{array}$ & $\begin{array}{c}\text { All Patients } \\
(\mathrm{n}=21)\end{array}$ \\
\hline Sex & $10(90.9)$ & $7(70)$ & $17(80.9)$ \\
\hline $\mathrm{F}$ & $1(9.1)$ & $3(30)$ & $4(19.1)$ \\
\hline $\mathrm{M}$ & 52.4 & 55.8 & 54.1 \\
\hline Mean age, yrs & $4(36.4)$ & $0(0)$ & $4(19)$ \\
\hline Former smoker & $2(18.2)$ & $5(50)$ & $7(33.3)$ \\
\hline Current smoker & $5(45.4)$ & $5(50)$ & $10(47.7)$ \\
\hline No history of smoking &
\end{tabular}

Values represent the number of patients (\%) unless indicated otherwise.

(Table 2). The mean procedure time and $\mathrm{CC}$ score were $247.80 \pm 55.49$ minutes and $2.30 \pm 0.48$, respectively, for the VR group. No statistical difference was found between the mean CC scores of the MCA aneurysms $(95 \%$ $\mathrm{CI}, \mathrm{p}=0.69)$. However, the mean procedure time was found to be significantly shorter, by 80.5 minutes $(95 \%$ $\mathrm{CI}, \mathrm{p}=0.012$ ), in the VR group compared with the nonVR control group. The VR group had a shorter mean procedure time for both unruptured and rupture aneurysms, although it was significantly shorter for unruptured aneurysms $(\mathrm{p}<0.01)$. Both groups had 4 patients with ruptured aneurysms with no significant difference in CC ( $p$ $>0.48$ ). The procedure time from the nonruptured aneurysms of the control and VR groups were compared with 2 years prior to the control group, from January 2014 to December 2015. The mean procedure time of this "precontrol" group $(\mathrm{n}=10)$ was not significantly different compared with the control group ( $p>0.6)$, yet it was significantly longer, by 63.8 minutes, when compared with the VR group $(\mathrm{p}<0.05)$.

Both groups were divided into subcategories according to their complexity level. The control group had at least 2 patients for each subcategory. In contrast, the VR group only comprised patients with CC scores of 2 and 3 . Therefore, subcategories for $\mathrm{CC}$ scores of 2 and 3 were further analyzed. In the control group, patients with $\mathrm{CC}$ scores of 2 and 3 had mean procedure times of $398.00 \pm 56.57 \mathrm{~min}-$ utes and $348.00 \pm 89.96$ minutes, respectively. The mean procedure times for patients with CC scores of 2 and 3 for the VR group were $236.14 \pm 52.01$ minutes and 275.00 \pm 64.49 minutes, respectively. Statistical significance was found between the procedure times for patients with a CC score of 2 between the two study groups $(95 \% \mathrm{CI}, \mathrm{p}=$ $0.0064)$. Other factors such as comorbidity were analyzed with no significant difference observed $(95 \% \mathrm{CI}, \mathrm{p}>0.05)$ between the two groups (Table 2). Furthermore, the CCI was found to have an insignificant difference between the two groups, although the VR group had a higher mean CCI than the control group (95\% CI, p = 0.14). Interestingly, the higher the CCI score the lower the estimated 10 -year survival. The CCI was not significantly different, yet there was a $15 \%$ difference in the estimated 10 -year survival between the VR and control groups (VR group, $72 \%$ vs control group, $87 \%$ ). The bottom line, accounting for these variables to eliminate other factors, is that the two groups displayed a uniform sample of CC scores and comorbidities prior to surgery.

No intraoperative complications occurred in any of the 21 patients, but each group reported complications during the postoperative hospital stay. In the control group, 1 patient experienced transient hemiparesis and aphasia thought to be due to vasospasm, with the hemiparesis resolving and the aphasia improving with treatment; the aphasia was better at 3 months and had resolved at 12 months. Two patients presented with a severe subarachnoid hemorrhage (SAH) with altered mental status, with one patient being hemiparetic and aphasic and the other with decreased level of consciousness (LOC) due to severe brain injury from intracranial hemorrhage and increased intracranial pressure. Both patients made improvements at 3 months and 1 year but remained severely disabled with modified Rankin Scale (mRS) scores of 3 and 5, respectively. In the VR group, one patient developed pneumonitis, and another with a subarachnoid hemorrhage (SAH) who experienced a generalized tonic-clonic seizure. However, symptoms resolved for both patients prior to discharge. Lastly, 1 patient in the VR group had a previous cerebrovascular accident (CVA) with right-sided hemiparesis and mild aphasia with an mRS score of 2 prior to surgery. Of these patients, none experienced new neurological deficits or a worsening of their mRS score directly related to the microsurgical clip occlusion of the aneurysm. The mean EBL and length of stay for the non-VR group were $254.54 \pm 121.36 \mathrm{ml}$ and $7.8 \pm 7.22$ days, respectively. The mean EBL and length of stay for the VR group were $260 \pm 126.49 \mathrm{ml}$ and $8.3 \pm$ 7.42 days, respectively (Table 3). The length of stay was longer for patients who experienced a ruptured aneurysm by approximately 10 days regardless of the planning group, which is expected given the need to monitor and treat the complications of an $\mathrm{SAH}$, including vasospasm, increased intracranial pressure, and hydrocephalus. The mean EBL

TABLE 2. Comparative mean values of procedure time, $\mathrm{CC}$ scores, and $\mathrm{CCl}$ scores

\begin{tabular}{lccc}
\hline \multicolumn{1}{c}{ Variable } & Control Group $(\mathrm{n}=11)$ & VR Group $(\mathrm{n}=10)$ & $\mathrm{p} \mathrm{Value}(95 \% \mathrm{Cl})$ \\
\hline Mean procedure time, min & $328.27 \pm 73.86$ & $247.80 \pm 55.49$ & 0.012 \\
\hline Ruptured aneurysm & $339 \pm 107.30(\mathrm{n}=4 ;$ CC 2.25$)$ & $277 \pm 42.91(\mathrm{n}=4 ;$ CC 2.5$)$ & $0.32 ;$ CC 0.67 \\
\hline Unruptured aneurysm & $322 \pm 56.75(\mathrm{n}=7 ;$ CC 2.57$)$ & $228 \pm 57.47(\mathrm{n}=6 ;$ CC 2.17) & $0.013 ;$ CC 0.48 \\
\hline Mean CC score & $2.45 \pm 1.13$ & $2.3 \pm 0.48$ & 0.69 \\
\hline Mean CCl score (\% 10-yr survival) & $1.8 \pm 1.17(87 \%)$ & $2.8 \pm 1.75(69 \%)$ & 0.14 \\
\hline
\end{tabular}

Mean values are presented as the mean $\pm S D$. 
TABLE 3. Comparison of the surgical complications, length of stay, and EBL during the procedure for non-VR and VR groups

\begin{tabular}{|c|c|c|}
\hline Variables & Control Group $(n=11)$ & VR Group $(n=10)$ \\
\hline \multirow{2}{*}{ On presentation } & $\begin{array}{l}\text { Hemiparesis \& aphasia due to ruptured aneurysm w/ } \\
\text { SAH \& IPH }(n=1)\end{array}$ & $\begin{array}{l}\text { CVA w/ mild aphasia, rt hemiparesis \& seizure disorder; prior to sur- } \\
\text { gery, patient diagnosed w/ aneurysm during stroke workup }(n=1)\end{array}$ \\
\hline & $\begin{array}{l}\text { Decreased LOC w/ SBI due to ruptured aneurysm, } \\
\text { required craniectomy }(n=1)\end{array}$ & Vasospasms \& speech difficulty in a patient w/ SAH $(n=1)$ \\
\hline \multirow{4}{*}{$\begin{array}{l}\text { Outcome on } \\
\text { discharge }\end{array}$} & $\begin{array}{l}\text { Hemiparesis \& aphasia due to ruptured aneurysm w/ } \\
\text { SAH \& IPH }(n=1)\end{array}$ & Pneumonitis; resolved prior to discharge $(n=1)$ \\
\hline & $\begin{array}{l}\text { Decreased LOC w/SBI due to ruptured aneurysm, } \\
\text { required craniectomy }(n=1)\end{array}$ & Single generalized seizure in a patient w/ SAH $(n=1)$ \\
\hline & & $\begin{array}{l}\text { CVA w/ mild aphasia, rt hemiparesis, \& seizure disorder; prior to sur- } \\
\text { gery, patient diagnosed w/ aneurysm during stroke work-up }(n=1)\end{array}$ \\
\hline & & Vasospasm \& mild aphasia in a patient w/ SAH $(n=1)$ \\
\hline \multirow{3}{*}{$\begin{array}{l}\text { Outcome } \\
\text { (at } 3 \text { mos) }\end{array}$} & Migraines $(n=1)$ & $\begin{array}{l}\text { CVA w/ mild aphasia, rt hemiparesis \& a seizure disorder; prior to sur- } \\
\text { gery, patient diagnosed w/ aneurysm during stroke workup }(n=1)\end{array}$ \\
\hline & $\begin{array}{l}\text { Hemiparesis \& aphasia due to ruptured aneurysm w/ } \\
\text { SAH \& IPH }(n=1)\end{array}$ & Mild aphasia in a patient w/ history of SAH $(n=1)$ \\
\hline & Decreased LOC $w / S B I$ due to ruptured aneurysm $(n=1)$ & \\
\hline \multirow{3}{*}{$\begin{array}{l}\text { Outcome } \\
\text { (at } 1 \mathrm{yr})\end{array}$} & Migraines improved w/ Botox $(n=1)$ & $\begin{array}{l}\text { CVA w/ mild aphasia, rt hemiparesis \& a seizure disorder; prior to sur- } \\
\text { gery, patient diagnosed w/ aneurysm during stroke workup }(n=1)\end{array}$ \\
\hline & Improved aphasia \& $r t$ sided weakness $(n=1)$ & Mild aphasia resolved in a patient $w /$ history of SAH $(n=1)$ \\
\hline & $\begin{array}{l}\text { Decreased LOC w/ SBI due to ruptured aneurysm; } \\
\text { developed seizures postcranioplasty, controlled by } \\
\text { medication, otherwise unchanged }(n=1)\end{array}$ & \\
\hline Mean LOS, days & $7.8 \pm 7.22$ & $8.3 \pm 7.42$ \\
\hline Mean EBL, ml & $254.54 \pm 121.36$ & $260 \pm 126.49$ \\
\hline
\end{tabular}

$\mathrm{IPH}=$ intraparenchymal hemorrhage; $\mathrm{LOS}$ = length of stay; $\mathrm{SBI}=$ severe brain injury.

Mean values are presented as the mean \pm SD.

was also higher by $130 \mathrm{ml}$ in patients with a ruptured aneurysm, regardless of which planning group they belonged.

\section{Discussion}

Even though VR is widely recognized for its potential application in surgical planning, the adoption for it is scarce, and as a result, limited data on its clinical impact exist. ${ }^{10,11}$ For aneurysm clipping, most reports on the value of preoperative planning in VR rely heavily on subjective feedback provided by the surgeons.$^{17-19}$ One study by Chugh et al. evaluated time per clip application and the overall time of clip placement for various types of aneurysms. ${ }^{11}$ The authors employed a previous version of the same Surgical Theater VR visualization platform used in the current study and found that fewer clip application attempts and decreased time per clip were associated with preoperative planning in conjunction with Sensable Technologies PHANTOM Omni haptic controllers and goggles. That particular study, however, did not take into account case complexity or clinical outcomes.

In this retrospective review, we report the utilization of the VR platform and Oculus VR headsets with controllers for preoperative planning and rehearsal of aneurysm clipping procedures using patient-specific 360VR models (Fig. 4) and its impact on total procedure time and clinical outcomes for patients with MCA aneurysms. CC and comorbidities were both taken into account. For each VR case the surgeon defined the location and size of the craniotomy using a virtual drill. For aneurysm clipping, various clip sizes and angles were rehearsed in VR. This allowed for the creation of a customized surgical plan, which has the potential to yield smaller craniotomies for MCA aneurysm clipping.

In our analysis, the mean procedure time for the VR group was significantly shorter than that in the control group, by approximately 80 minutes. This is not only statistically but clinically significant, meaning it is not simply a reduction in OR time by a few minutes but by nearly $25 \%$. Reduction in procedure time has been associated with decreased time that patients are under general anesthesia and lessening of associated risks that include heart attack, blood pressure changes, venous thromboembolism, or stroke..$^{20-24}$ This is especially true of patients who have experienced a ruptured aneurysm, who are critically ill and may be in pulmonary failure, or who have cardiac stun or a myriad of other complications due to an SAH. Furthermore, shorter procedure times result in increased workflow efficiency. Several cost analysis breakdowns highlight that OR time is one of biggest expenses during a patient's surgical hospital stay. ${ }^{20,21}$ Therefore, decreasing procedure time and consequently total OR time may result in better allocation of resources. ${ }^{25,26}$ Additionally, more staff and OR space would be available to care for a greater number of patients. 

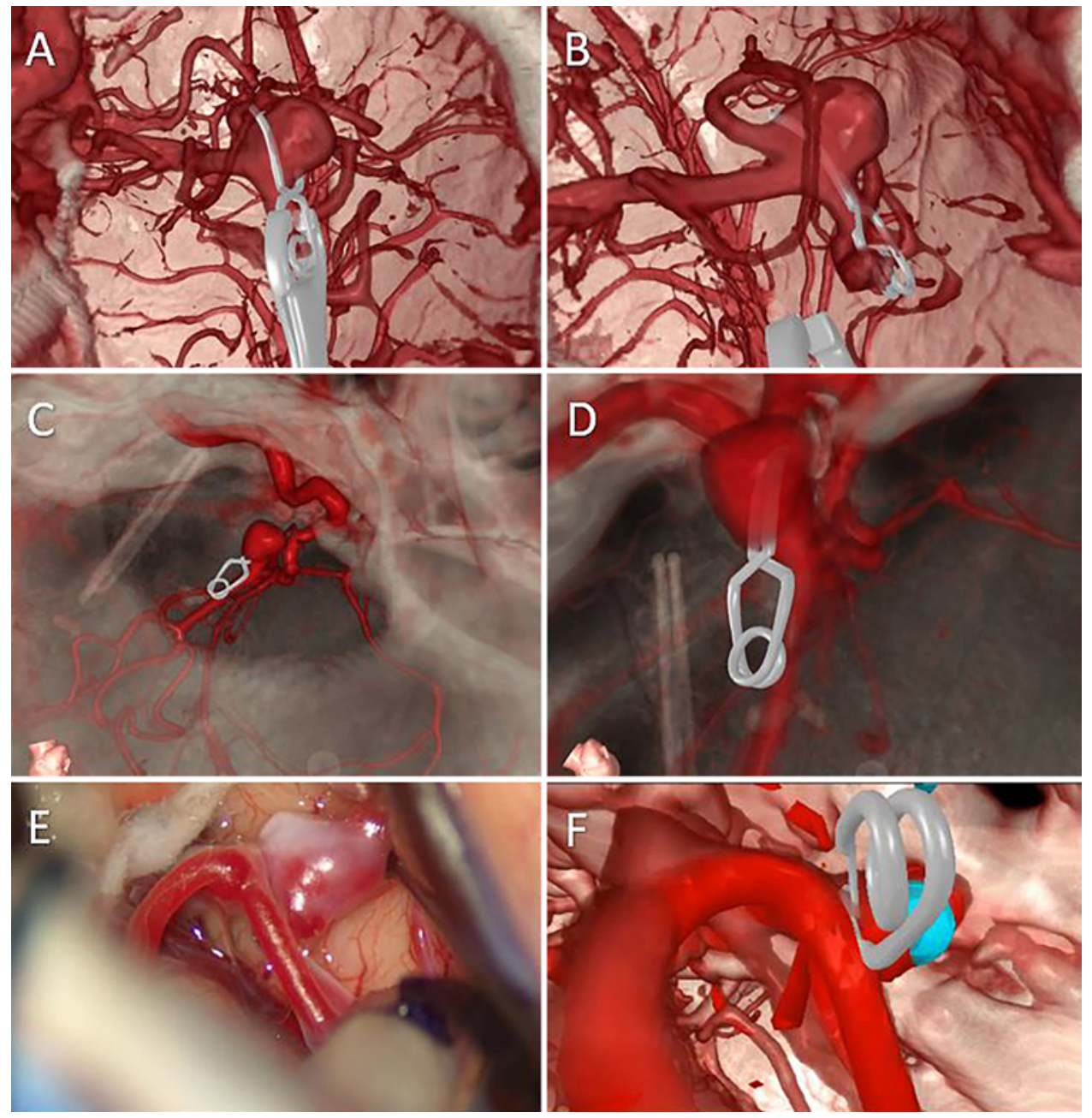

FIG. 4. Snapshots of 360VR models and intraoperative images of aneurysm clip placements. A and B: Snapshots of a virtual clip placement within the 360VR model prior to surgery. $C$ and D: OR reference images displayed during the surgery for craniotomy placement (C) and clip placement (D). E and F: Intraoperative image of an MCA aneurysm alongside the corresponding snapshot of the 360VR model with the virtual clip and marker (blue) designating the location of the MCA aneurysm.

Even though there was a significant reduction in procedure time in the VR group, clinical outcomes were not affected, as expected, due to the experience and expertise of the surgeon. Preoperative planning in VR may prove more beneficial for less-experienced surgeons. Interactive 3D and VR models have been implemented in neurosurgery mainly as resources for surgical education and training, finding greater benefits for trainees. ${ }^{27-29}$ Another aspect that was analyzed was the difference in overall patient health in each study group prior to treatment. No significant differences were observed in the CCI scores between the two groups; therefore, the morbidity, mortality, and risks of complication were similar. ${ }^{18,30}$

From this study, it is clear that using VR for preoperative planning and rehearsal mainly for unruptured aneurysm cases significantly decreased the operative time (Table 1); however, the underlying reasons are not as clear. By better understanding the patient's anatomy, namely, visualizing the vascular anatomy in 3D and being able to conceptualize it in the context of the skull, significantly improves planning of the approach and positioning of the patient. Also, having planned out and rehearsed gaining proximal control, dissection of the aneurysm, and microsurgical clipping subjectively increases confidence and allows for a more assertive, time-efficient approach to the aneurysm. Although these data were not captured, subjectively and in the senior author's experience, nearly $75 \%$ of the time that the clips were used during the VR preoperative rehearsal were applied during surgery. Prior to the use of Surgical Theater, perhaps $50 \%$ of the time the correct clips were identified prior to the procedure. Although all the above factors contribute to increased efficiency, it is difficult to prove or assign which of them contributes most to the equation. A confounding variable that could affect the operative time is the improved performance of the surgeon by gained experience over time. It is generally accepted that a surgeon's efficiency and expertise evolve throughout their career. Since the surgeon in this study had 13 years of experience prior to the control group, we predicted the improvement would be modest at best. To address this con- 
cern, the authors analyzed the elective cases from 2 years prior to the control group, from January 2014 to December 2015. When the procedure times of this "precontrol group" were compared with the control group, there was no significant difference. However, similar to the findings when comparing the procedure times for the control group with the VR group, there was a significant decrease in procedure times comparing the "precontrol group" with the VR group $(\mathrm{p}<0.05)$.

This study was limited by the lack of an agreed on and validated complexity scoring system to indicate the difficulty of surgically treating a particular aneurysm. Experts will generally agree that increased size of an aneurysm and the width of the neck with the need to reconstruct the parent vessel, as well as distal vessels being incorporated into the aneurysm, increase the complexity and difficulty of surgical treatment. Additionally, the rate of clip correction and occlusion rates were not compared. Future studies with a larger sample size and multiple surgeons with different levels of experience over the same period of time should be conducted to corroborate the trends found in this study. The successful rate of predicting the correct clip and trajectory for both the traditional and VR preoperative planning can also be analyzed and compared. As discussed earlier, surgeons with less experience may benefit more from this technology. The study can also be expanded to evaluate other types of aneurysms or neurovascular pathologies. Further evaluation on the differences between ruptured and unruptured aneurysms should also be assessed.

\section{Conclusions}

Patient-specific 360VR models were used for planning the craniotomy and rehearsing with various clip sizes and configurations in a VR group and a non-VR control group. The significant difference in procedure time between the two groups shows that VR enhances surgical efficiency for MCA aneurysm clipping procedures, which is likely generalizable to microsurgical treatment of all intracranial aneurysms. Decreased operative times have important implications and likely will lead to decreased overall complications due to reducing anesthesia time, as well as reducing treatment costs and improving efficiency.

\section{References}

1. Morita A, Kirino T, Hashi K, Aoki N, Fukuhara S, Hashimoto N, et al. The natural course of unruptured cerebral aneurysms in a Japanese cohort. N Engl J Med. 2012;366(26): 2474-2482.

2. Rinne J, Hernesniemi J, Niskanen M, Vapalahti M. Analysis of 561 patients with 690 middle cerebral artery aneurysms: anatomic and clinical features as correlated to management outcome. Neurosurgery. 1996;38(1):2-11.

3. Spetzler RF, McDougall CG, Albuquerque FC, Zabramski JM, Hills NK, Partovi S, et al. The Barrow Ruptured Aneurysm Trial: 3-year results. J Neurosurg. 2013;119(1):146-157.

4. Bakker NA, Metzemaekers JD, Groen RJ, Mooij JJ, Van Dijk JM. International subarachnoid aneurysm trial 2009: endovascular coiling of ruptured intracranial aneurysms has no significant advantage over neurosurgical clipping. Neurosurgery. 2010;66(5):961-962.

5. Molyneux AJ, Kerr RSC, Yu LM, Clarke M, Sneade M, Yar- nold JA, Sandercock P. International subarachnoid aneurysm trial (ISAT) of neurosurgical clipping versus endovascular coiling in 2143 patients with ruptured intracranial aneurysms: a randomised comparison of effects on survival, dependency, seizures, rebleeding, subgroups, and aneurysm occlusion. Lancet. 2005;366(9488):809-817.

6. Yang W, Huang J. Treatment of middle cerebral artery (MCA) aneurysms: a review of the literature. Chin Neurosurg $J$. Published online August 18, 2015. doi:10.1186/s41016-0150001-8

7. Stadie AT, Kockro RA, Serra L, Fischer G, Schwandt E, Grunert P, Reisch R. Neurosurgical craniotomy localization using a virtual reality planning system versus intraoperative image-guided navigation. Int J CARS. 2011;6(5):565-572.

8. Neyazi B, Saalfeld P, Berg P, Skalej M, Preim B, Sandalcioglu IE, Saalfeld S. VR craniotomy for optimal intracranial aneurysm surgery planning. Paper presented at: Computer and Robotic Assisted Surgery (CURAC); September 2019; Reutlingen, Germany. Accessed June 8, 2021. https://www. researchgate.net/publication/335927598_VR_Craniotomy_ for_Optimal_Intracranial_Aneurysm_Surgery_Planning

9. Mori K, Esaki T, Yamamoto T, Nakao Y. Individualized pterional keyhole clipping surgery based on a preoperative three-dimensional virtual osteotomy technique for unruptured middle cerebral artery aneurysm. Minim Invasive Neurosurg. 2011;54(5-6):207-213.

10. Bambakidis NC, Selman WR, Sloan AE. Surgical rehearsal platform: potential uses in microsurgery. Neurosurgery. 2013; 73(suppl 1):122-126.

11. Chugh AJ, Pace JR, Singer J, Tatsuoka C, Hoffer A, Selman WR, Bambakidis NC. Use of a surgical rehearsal platform and improvement in aneurysm clipping measures: results of a prospective, randomized trial. J Neurosurg. 2017;126(3):838844.

12. Ferroli P, Tringali G, Acerbi F, Schiariti M, Broggi M, Aquino D, Broggi G. Advanced 3-dimensional planning in neurosurgery. Neurosurgery. 2013;72(suppl 1):54-62.

13. Pelargos PE, Nagasawa DT, Lagman C, Tenn S, Demos JV, Lee SJ, et al. Utilizing virtual and augmented reality for educational and clinical enhancements in neurosurgery. J Clin Neurosci. 2017;35:1-4.

14. Clark AD, Barone DG, Candy N, Guilfoyle M, Budohoski K, Hofmann R, et al. The effect of 3-dimensional simulation on neurosurgical skill acquisition and surgical performance: a review of the literature. J Surg Educ. 2017;74(5):828-836.

15. Quan H, Li B, Couris CM, Fushimi K, Graham P, Hider P, et al. Updating and validating the Charlson comorbidity index and score for risk adjustment in hospital discharge abstracts using data from 6 countries. Am J Epidemiol. 2011;173(6): 676-682.

16. Etminan N, Brown RD Jr, Beseoglu K, Juvela S, Raymond J, Morita A, et al. The unruptured intracranial aneurysm treatment score: a multidisciplinary consensus. Neurology. 2015; 85(10):881-889.

17. Haridas A, Miller M. Middle cerebral artery aneurysm clipping with immersive $360^{\circ}$ virtual reality model: 2 -dimensional operative video. Oper Neurosurg (Hagerstown). 2021; 20(4):E314.

18. Kockro RA, Killeen T, Ayyad A, Glaser M, Stadie A, Reisch $\mathrm{R}$, et al. Aneurysm surgery with preoperative three-dimensional planning in a virtual reality environment: technique and outcome analysis. World Neurosurg. 2016;96:489-499.

19. Zawy Alsofy S, Sakellaropoulou I, Nakamura M, Ewelt C, Salma A, Lewitz M, et al. Impact of virtual reality in arterial anatomy detection and surgical planning in patients with unruptured anterior communicating artery aneurysms. Brain Sci. 2020;10(12):E963.

20. Liu J, Gormley N, Dasenbrock HH, Aglio LS, Smith TR, Gormley WB, Robertson FC. Cost-benefit analysis of 
transitional care in neurosurgery. Neurosurgery. 2019;85(5): 672-679.

21. McLaughlin N, Upadhyaya P, Buxey F, Martin NA. Valuebased neurosurgery: measuring and reducing the cost of microvascular decompression surgery. J Neurosurg. 2014; 121(3):700-708.

22. Chen WK, Ren L, Wei Y, Zhu DX, Miao CH, Xu JM. General anesthesia combined with epidural anesthesia ameliorates the effect of fast-track surgery by mitigating immunosuppression and facilitating intestinal functional recovery in colon cancer patients. Int J Colorectal Dis. 2015; 30(4):475-481.

23. Clarke-Pearson DL, Dodge RK, Synan I, McClelland RC, Maxwell GL. Venous thromboembolism prophylaxis: patients at high risk to fail intermittent pneumatic compression. Obstet Gynecol. 2003;101(1):157-163.

24. Kim JY, Khavanin N, Rambachan A, McCarthy RJ, Mlodinow AS, De Oliveria GS Jr, et al. Surgical duration and risk of venous thromboembolism. JAMA Surg. 2015;150(2):110117.

25. Doyle LA, Yondorf M, Peng C, Harrison AS, Den RB. Process mapping and time study to improve efficiency of new procedure implementation for high-dose rate prostate brachytherapy. J Healthc Qual. 2018;40(1):19-26.

26. Macario A. What does one minute of operating room time cost? J Clin Anesth. 2010;22(4):233-236.

27. Alaraj A, Luciano CJ, Bailey DP, Elsenousi A, Roitberg BZ, Bernardo A, et al. Virtual reality cerebral aneurysm clipping simulation with real-time haptic feedback. Neurosurgery. 2015;11(suppl 2):52-58.

28. Stepan K, Zeiger J, Hanchuk S, Del Signore A, Shrivastava $\mathrm{R}$, Govindaraj S, Iloreta A. Immersive virtual reality as a teaching tool for neuroanatomy. Int Forum Allergy Rhinol. 2017;7(10):1006-1013.

29. Tucker AM, Beckett JS, Martin NA. Next generation case report: supraorbital craniotomy for anterior communicating artery aneurysm clipping in annotated virtual reality environment. Oper Neurosurg (Hagerstown). 2018;15(5):E73-E76.
30. Dehdashti AR, Mermillod B, Rufenacht DA, Reverdin A, de Tribolet N. Does treatment modality of intracranial ruptured aneurysms influence the incidence of cerebral vasospasm and clinical outcome? Cerebrovasc Dis. 2004;17(1):53-60.

\section{Disclosures}

Daniela Barbery is employed by Surgical Theater; she is a fulltime field specialized technician at the Neuroscience Institute at JFK Medical Center that provides technical support for all clinical cases.

\section{Author Contributions}

Conception and design: both authors. Acquisition of data: both authors. Analysis and interpretation of data: both authors. Drafting the article: both authors. Critically revising the article: both authors. Reviewed submitted version of manuscript: both authors. Approved the final version of the manuscript on behalf of both authors: Steineke. Administrative/technical/material support: Steineke. Study supervision: Steineke.

\section{Supplemental Information \\ Videos}

Video 1. https://vimeo.com/558659933.

\section{Previous Presentations}

Portions of this work were submitted and accepted in abstract form for the CNS 2020 Annual Meeting, November 16, 2020.

\section{Correspondence}

Thomas C. Steineke: JFK Neuroscience Institute, Edison, NJ. thomas.steineke@hmhn.org. 